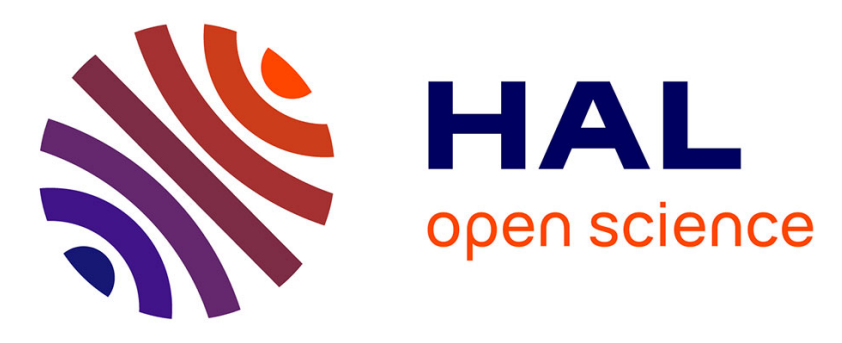

\title{
Reliability-based design optimization of shank chisel plough using optimum safety factor strategy
}

G. Kharmanda, Mohamed Ibrahim, A. Abo Al-Kheer, Fabrice Guérin, Abdelkhalak El Hami

\section{- To cite this version:}

G. Kharmanda, Mohamed Ibrahim, A. Abo Al-Kheer, Fabrice Guérin, Abdelkhalak El Hami. Reliability-based design optimization of shank chisel plough using optimum safety factor strategy. Computers and Electronics in Agriculture, 2014, 109, Non spécifié. 10.1016/j.compag.2014.09.001 . hal-03287226

\section{HAL Id: hal-03287226 \\ https://univ-angers.hal.science/hal-03287226}

Submitted on 15 Jul 2021

HAL is a multi-disciplinary open access archive for the deposit and dissemination of scientific research documents, whether they are published or not. The documents may come from teaching and research institutions in France or abroad, or from public or private research centers.
L'archive ouverte pluridisciplinaire HAL, est destinée au dépôt et à la diffusion de documents scientifiques de niveau recherche, publiés ou non, émanant des établissements d'enseignement et de recherche français ou étrangers, des laboratoires publics ou privés. 


\title{
Reliability-based design optimization of shank chisel plough using optimum safety factor strategy
}

\author{
G. Kharmanda ${ }^{\mathrm{a}, *}, \mathrm{M}-\mathrm{H}$. Ibrahim ${ }^{\mathrm{b}}$, A. Abo Al-kheer ${ }^{\mathrm{c}}$, F. Guerin ${ }^{\mathrm{b}}$, A. El-Hami ${ }^{\mathrm{d}}$ \\ a Division of Solid Mechanics, Lund University, Lund, Sweden \\ ${ }^{\mathrm{b}}$ Ecole d'Ingénieurs de l'Université d'Angers, Angers, France \\ ${ }^{\mathrm{c}}$ Faculty of Agricultural Engineering, University of Aleppo, Aleppo, Syria \\ ${ }^{\mathrm{d}}$ INSA de Rouen, LOFIMS, St Etienne du Rouvray, France
}

\section{A R T I C L E I N F O}

\section{Article history:}

Received 4 June 2014

Received in revised form 27 July 2014

Accepted 10 September 2014

\section{Keywords}

Design optimization

Structural reliability

Random variables

Soil properties

Soil tillage equipment

Optimum safety factors

\begin{abstract}
A B S T R A C T
Reliability integration into tillage machine design process is a new strategy to overcome the drawbacks of classical design approaches and to achieve designs with a required reliability level. Furthermore, design optimization of soil tillage equipments under uncertainty seeks to design structures which should be both economic and reliable. The originality of this research is to develop an efficient methodology that controls the reliability levels for complex statistical distribution cases of random tillage forces. This developed strategy is based on design sensitivity concepts in order to determine the influence of each random parameter. The application of this method consists in taking into account the uncertainties on the soil tillage forces. The tillage forces are calculated in accordance with analytical model of McKyes and Ali with some modifications to include the effect of both soil-metal adhesion and tool speed. The different developments and applications show the importance of the developed method to improve the performance of the soil tillage equipments considering both random geometry and loading parameters. The developed method so-called OSF (Optimum Safety Factor) can satisfy a required reliability level without additional computing time relative to the deterministic design optimization study. Since the agricultural equipment parameters are extremely nonlinear, we extended the OSF approach to several nonlinear probabilistic distributions such as lognormal, uniform, Weibull and Gumbel probabilistic distribution laws.
\end{abstract}

(c) 2014 Elsevier B.V. All rights reserved.

\section{Introduction}

In the deterministic design optimization (Arora, 1989; Haftaka and Gurdal, 1991), the designer aims to reduce the engineering design cost without caring about the effects of uncertainties concerning materials, geometry and loading. The resulting optimal solution may therefore represent an inappropriate reliability level. However, the integration of reliability analysis during the optimization process leads to reduce the structural weight in uncritical regions that does not only provide an improved design but also a higher level of confidence in the design. This model is called Reliability-Based Design Optimization (RBDO). Here, we distinguish three approach families: Coupled, Decoupled and Single Loop Approaches. The classical coupled approach can be carried out in two separate spaces: the physical space and the normalized space

\footnotetext{
* Corresponding author.

E-mail address: mgk@scs-net.org (G. Kharmanda).
}

(two nested optimization problems). Since many repeated searches are needed in the above two spaces, the computational time for such an optimization is a big problem. The solution of the above nested problems leads to very high computational cost, especially for large-scale structures (Feng and Moses, 1986). The major difficulty lies in the structural reliability evaluation, which is carried out by a special optimization procedure. The decoupled approach such as SORA (Sequential Optimization and Reliability Assessment) is carried out in two successive loops (Du and Chen, 2004). In order to improve the numerical performance, a single loop approach such as OSF (Optimum Safety Factor) can be efficiently applied on linear cases (Kharmanda et al., 2009). The distributions of soil-tool forces are established to design soil tillage equipment such as shank chisel plough (Abo Al-kheer, 2010). In this paper, the OSF method is extended to several nonlinear probabilistic distributions. An efficient method is developed based on the optimality conditions. In this work, we use a statistical study of the soil tillage forces, based on soil property randomness. 


\section{Mechanical properties of soil}

The working part of tillage equipment (ex: plow bottoms in moldboard plows, disk blades in disk plows) receiving energy from the tractor or other source works the soil and changes its state and properties. To determine the influence between the soil and tillage tool, we should determine the distribution type for each soil mechanical properties. The mechanical properties of soil which are important during soil-working, that is, properties which influence the nature of the process, hence the properties which have effects on the forces acting on the tillage tool are: Soil bulk density, Angle of internal friction, Angle of external friction, Cohesion and Adhesion. We elaborated a table of 32 samples of the above five mechanical properties of soils from our previous work (Abo Al-kheer, 2010). This data presented in Appendix, can be helpful to establish the soil tillage force probabilistic model.

\section{Soil tillage forces}

Many methods and models had been used to predict the forces acting on the tillage tool. However, the majority of researchers have used the general earth pressure model, proposed by Reece, 1965. The total force acting on the tillage tool can be written as follows:

$P=P_{\gamma}+P_{c}+P_{c a}+P_{q}+P_{a}$

Here, $P$ is the total soil cutting force acting on the tillage tool $(\mathrm{kN})$, $P_{\gamma}$ is the force acting on the tillage tool caused by soil gravity $(\mathrm{kN})$, $P_{c}$ is the force acting on the tillage tool caused by cohesion $(\mathrm{kN}), P_{c a}$ is the force acting on the tillage tool caused by adhesion $(\mathrm{kN}), P_{q}$ is the force acting on the tillage tool caused by surcharge pressure $(\mathrm{kN})$ and $P_{a}$ is the force acting on the tillage tool caused by tool speed $(\mathrm{kN})$.

In our work McKyes and Ali's model (1977), as shown in Fig. 1, was used to estimate the forces acting on a tillage tool with three main modifications (Abo Al-kheer et al., 2011). The effects of soil-tool adhesion and tool speed were taken into account. The total force can be written according to the Equation (1a) as:

$P=\left(\gamma d^{2} N_{\gamma}+c d N_{c}+c_{a} d N_{c a}+q d N_{q}+\gamma v^{2} d N_{a}\right) w$

where $\gamma$ is the soil specific weight in $\left(\mathrm{kN} / \mathrm{m}^{-3}\right), d$ is the tool working depth in (m), $N_{\gamma}$ is the gravity coefficient (dimensionless), $c$ is the soil cohesion in ( $\mathrm{kPa}), N_{c}$ is the cohesion coefficient (dimensionless), $c_{a}$ is the soil-tool adhesion in ( $\left.\mathrm{kPa}\right), N_{c a}$ is the adhesion coefficient (dimensionless), $q$ is the surface surcharge pressure in ( $\mathrm{kPa}), N_{q}$ is the surcharge pressure coefficient (dimensionless), $v$ is the tool speed in $(\mathrm{m} / \mathrm{s}), N_{a}$ is the inertial coefficient (dimensionless) and $w$ is the tool width in (m). Dimensionless coefficients $\left(N_{\gamma}, N_{c}, N_{c a}, N_{q}\right.$, $N_{a}$ ) can be determined with respect to the soil failure pattern proposed by McKyes and Ali (1977).

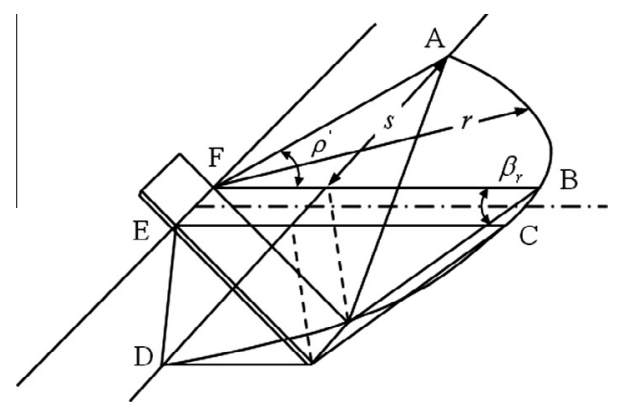

Fig. 1. Soil failure model for narrow blades, after McKyes and Ali (1977).
Furthermore, the width of the side crescent was calculated using an empirical regression equation and the rupture angle $\beta_{r}$ was obtained by minimizing the total force.

The horizontal and vertical forces were calculated using the following two equations, respectively:

$P_{H}=P \sin (\alpha+\delta)+c_{a} d w \cos (\alpha)$

$P_{V}=P \cos (\alpha+\delta)-c_{a} d w$

where $P_{H}$ is the horizontal force in $(\mathrm{kN})$ and $P_{V}$ is the vertical force in $(\mathrm{kN})$. According to Eqs. (1) and (2), the tillage system parameters considered for the calculation of the horizontal and vertical forces can be grouped into three main categories: soil engineering properties, tool design parameters and operational conditions.

\section{Structural reliability}

In structural reliability theory many effective techniques have been developed during the last 40 years to estimate the reliability, namely FORM (First Order Reliability Methods), SORM (Second Order Reliability Method) and simulation techniques (Hasofer and Lind, 1974). Here, we consider two kinds of variables: Design variables and Random variables. The image of the random variables in the standard normalized space is denoted $\mathbf{u}$, calculated by: $\mathbf{u}=T(\mathbf{y})$ where $T(\mathbf{y})$ is the probabilistic transformation function (Fig. 2). For a given failure scenario, the reliability index $\beta$ is evaluated by solving a constrained minimization problem:

$\beta=\min d(\mathbf{u}) \quad$ subject to : $H(\mathbf{u})=0$

with

$d=\sqrt{\sum u_{i}^{2}}$

where $\mathbf{u}$ is the vector modulus in the normalized space, measured from the origin see Fig. 2.

The solution to problem (3) defines the Most Probable failure Point (MPP), see Fig. 2. The resulting minimum distance between the limit state function $H(\mathbf{u})$ and the origin, is called the reliability index $\beta$. The results are subjected to classical difficulties in nonlinear programming: existence of local minima, gradient approximation and computational time. The random variables are assembled in the vector $\mathbf{y}$ and represent the structural uncertainties which are identified by probabilistic distributions. These variables can be geometrical dimensions, material characteristics or applied external loading (Hasofer and Lind, 1974).

\section{Reliability-Based Design Optimization (RBDO)}

Traditionally, for the reliability-based optimization procedure we use two spaces: the physical space and the normalized space see Lemaire, 2005. Therefore, the reliability-based optimization is performed by nesting the following two problems:

Problem I: Optimization problem: this problem seeks to minimize an objective function subject to deterministic constraints and reliability requirements which is defined as follows:

$$
\begin{array}{ll}
\min & f(\mathbf{x}) \\
\text { subject to } & g_{k}(\mathbf{x}) \leq 0, \quad k=1, \ldots, K \\
\text { and } & \beta(\mathbf{x}, \mathbf{u}) \geq \beta_{t}
\end{array}
$$

where $f(\mathbf{x})$ is the objective function, $g_{k}(\mathbf{x}) \leqslant 0$ are the associated constraints, $\beta(\mathbf{x}, \mathbf{u})$ is the reliability index of the structure, and $\beta_{t}$ is the target reliability index.

Problem II: Reliability analysis: the reliability index $\beta(\mathbf{x}, \mathbf{u})$ is the minimum distance between the limit state function $H(\mathbf{x}, \mathbf{u})$ and the 


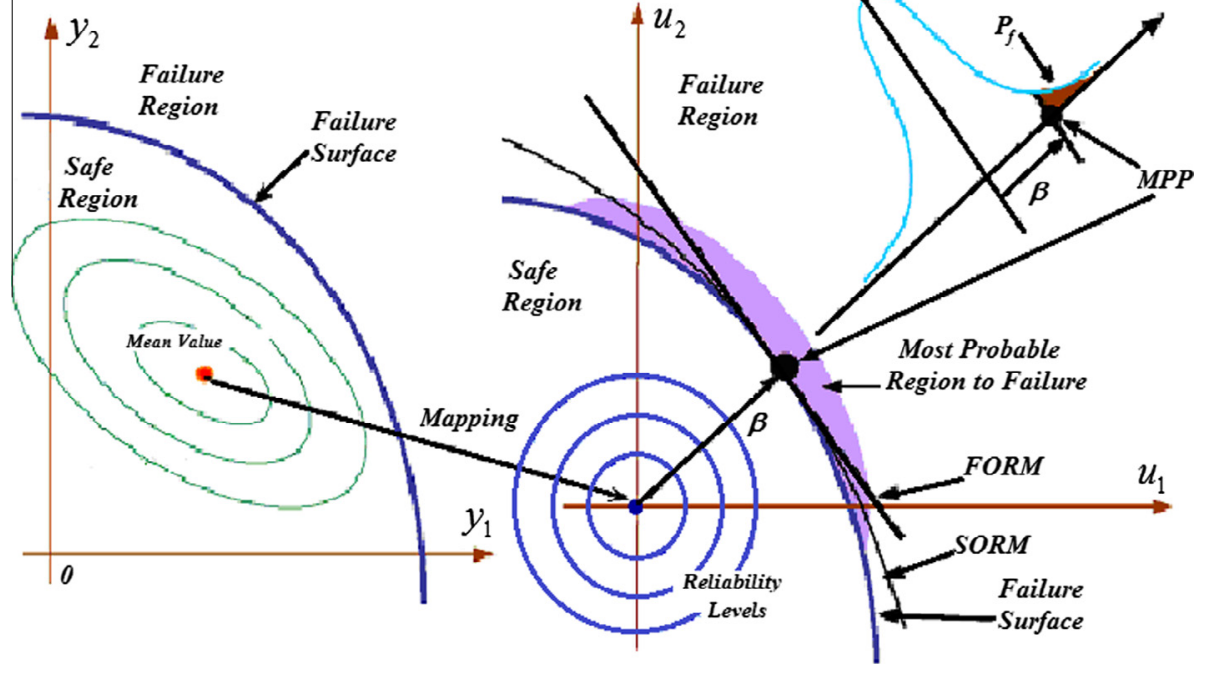

Fig. 2. Physical and normalized spaces.

origin, see Fig. 2. This index is determined by solving the minimization problem:

$\min \quad d(\mathbf{u})$

subject to $H(\mathbf{x}, \mathbf{u}) \leqslant 0$

where $d(\mathbf{u})$ is the distance in the normalized random space, given by $d=\sqrt{\sum u_{i}^{2}}$, and $H(\mathbf{x}, \mathbf{u})$ is the performance function (or limit state function) in the normalized space, defined such that $H(\mathbf{x}, \mathbf{u}) \leqslant 0$ implies failure, see Fig. 2 . In the physical space, the image of $H(\mathbf{x}, \mathbf{u})$ is the limit state function $G(\mathbf{x}, \mathbf{y})$, see Fig. 2. Using the classical approach, the RBDO process is carried out in two spaces and leads to a high computational time problem. A hybrid approach based on simultaneous solution of the reliability and the optimization problem is developed. This approach consists in minimizing a new form of the objective function $F(\mathbf{x}, \mathbf{y})$ subject to a limit state and to deterministic as well as to reliability constraints:

$\min$

subject to

$$
F(\mathbf{x}, \mathbf{y})=f(\mathbf{x}) \cdot d_{\beta}(\mathbf{x}, \mathbf{y})
$$

and

$$
G(\mathbf{x}, \mathbf{y}) \leq 0
$$$$
g_{k}(\mathbf{x}) \leq 0, \quad k=1, \ldots, K
$$

Here, $d_{\beta}(\mathbf{x}, \mathbf{y})$ is the distance in the hybrid space between the optimum and the design point, $d_{\beta}(\mathbf{x}, \mathbf{y})=d(\mathbf{u})$. The minimization of the function $F(\mathbf{x}, \mathbf{y})$ is carried out in the Hybrid Design Space (HDS) of deterministic variables $\mathbf{x}$ and random variables $\mathbf{y}$ (Kharmanda et al., 2009).

\section{Optimum safety factor developments}

\subsection{Formulation developments}

At the MPP, $\mathbf{u}^{*}$, is the solution of the Karush-Kuhn-Tucker (KKT) conditions of the FORM optimization problem (3).

$u_{i}^{*}=-\beta \frac{\frac{\partial H}{\partial u_{i}}}{\left\|\frac{\partial H}{\partial u_{i}}\right\|}$

The derivative of the limit state $H$ with respect to $\mathbf{u}$ and the derivative of $\mathbf{u}$ with respect to the design variable $\mathbf{x}$ can be expanded in terms of the original random variables $\mathbf{y}$ as follows: $\frac{\partial H}{\partial u_{i}}=\frac{\partial G}{\partial y_{k}} \frac{\partial T_{k}^{-1}(\mathbf{u})}{\partial u_{i}}$ and $\frac{\partial \mathbf{u}^{*}}{\partial x_{i}}=\frac{\partial T_{k}(\mathbf{u})}{\partial y_{k}} \frac{\partial x^{*}}{\partial y_{k}}$

For simplicity, consider now the case of $n$ normalized variables $u_{i}, i=1, \ldots, n$ (see Fig. 3: two normalized variables $u_{1}$ and $u_{2}$ ). For an assumed failure scenario, we define $H(\mathbf{u}) \leqslant 0$ and $G(\mathbf{y}) \leqslant 0$ as limit state functions in the normalized space (u-space) and in physical one ( $\mathbf{y}$-space). Here, the design point $P^{*}$ can be calculated by

$\min : \quad d^{2}=u_{1}^{2}+u_{2}^{2}+\cdots+u_{n}^{2}$

subject to : $H\left(u_{1}, u_{2}, \ldots, u_{n}\right) \leqslant 0$

The Lagrangian function for the problem (9) can be written as

$L(\mathbf{u}, \lambda, s)=d^{2}(\mathbf{u})+\lambda \cdot\left[H(\mathbf{u})+s^{2}\right]$

where the inequality constraint in (9) is adjoined by means of the Lagrange multiplier $\lambda$, after having converted the inequality constraint into the equality $H(\mathbf{u})+s^{2}=0$ by introducing the real slack variable $s$. The optimality conditions for the Lagrangian are:

$\frac{\partial L}{\partial u_{i}}=\frac{\partial d^{2}}{\partial u_{i}}+\lambda \frac{\partial H}{\partial u_{i}}=0, \quad i=1, \ldots, n$

$\frac{\partial L}{\partial \lambda}=H(\mathbf{u})+s^{2}=0$

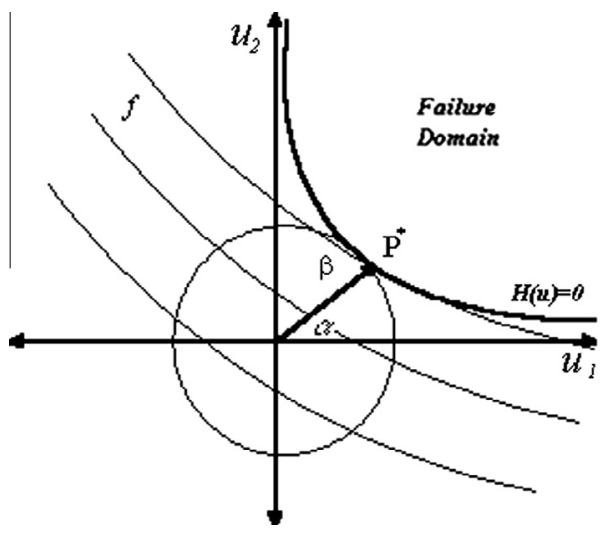

Fig. 3. Design point with two normalized variables. 
$\frac{\partial L}{\partial s}=2 s \lambda=0$

The optimality condition for $L$ with respect to $s$ yields the so-called switching condition $s \lambda=0$, and the necessary condition $\partial^{2} L / \partial s^{2} \geqslant 0$ for a minimum of $L$ implies that the Lagrangian multiplier $\lambda$ must be non-negative, i.e., $\lambda \geqslant 0$. Due to condition (11c), we can distinguish between two cases:

Case 1: If the real slack variable is non-zero $(s \neq 0)$, the Lagrangian multiplier has to be zero $(\lambda=0)$ and the limit state function must be less than zero $(H(\mathbf{u})<0)$, which corresponds to the case of safety.

Case 2: If the real slack variable is zero $(s=0)$, the Lagrangian multiplier is non-negative $(\lambda \geqslant 0)$ and the limit state is defined by the equality constraint $H(\mathbf{u})=0$. The solution here is found on the limit state surface and represents the Design Point.

The first case is not suitable to our reliability-based study whereas the second one is basic for our approach. Using the expression for the square distance $d^{2}$ given by Eq. (9) and by introducing in (11a), we get:

$u_{i}=-\frac{\lambda}{2} \frac{\partial H}{\partial u_{i}}, \quad i=1, \ldots, n$

Problem (9) gives us the reliability index $\beta$ as the minimum distance between the limit state surface and the origin. This means that the resulting reliability index may be lower or higher than the target reliability index $\beta_{t}$. As we seek to satisfy a required target reliability level for the optimization problem, we can write

$\beta_{t}^{2}=\sum_{i=1}^{n} u_{i}^{2}$

To determine $\lambda$ in (12), we now substitute index $i$ by $j$ in (12), square both sides of the equation, and sum from $j=1$ to $n$. Using (13), we then obtain

$$
\left(\frac{\lambda}{2}\right)^{2}=\frac{\beta_{t}^{2}}{\sum_{j=1}^{n}\left(\frac{\partial H}{\partial u_{j}}\right)^{2}}
$$

which upon substitution into (12) yields the following expression for the normalized variable $u_{i}$,

$u_{i}= \pm \beta_{t} \sqrt{\frac{\left(\frac{\partial H}{\partial u_{i}}\right)^{2}}{\sum_{j=1}^{n}\left(\frac{\partial H}{\partial u_{j}}\right)^{2}}}$

Eq. (15) at the optimum value of the normalized vector can be written in the following form:

$u_{i}^{*}= \pm \beta_{t} \sqrt{\frac{\left(\frac{\partial H}{\partial u_{i}}\right)^{2}}{\sum_{j=1}^{n}\left(\frac{\partial H}{\partial u_{j}}\right)^{2}}}$

where the sign of \pm depends on the sign of the derivative, i.e.,

$\frac{\partial G}{\partial y_{i}}>0 \Longleftrightarrow u_{i}^{*}>1$ and $\frac{\partial G}{\partial y_{i}}<0 \Longleftrightarrow u_{i}^{*}<1$

The calculation of the normalized gradient $\partial H / \partial \mathbf{u}$ is not directly accessible because the mechanical analysis is carried out in the physical space rather than in the standard space. However, using theory of statistics we can derive the following expression from which the computation of the normalized gradient can be carried out by applying the chain rule on the physical gradient $\partial G / \partial \mathbf{y}$ :

$\frac{\partial H}{\partial u_{i}}=\frac{\partial G}{\partial y_{k}} \frac{\partial T_{k}^{-1}(\mathbf{u})}{\partial u_{i}}, \quad i=1, \ldots, n, k=1, \ldots, K$ where $T^{-1}(\mathbf{u})$ denotes the inverse mapping of $\mathbf{u}=T(\mathbf{y})$ from standard normalized space $\mathbf{u}$ into the random space $\mathbf{y}$. It is not easy to find the derivative of the inverted probabilistic transformation function $T^{-1}(\mathbf{u})$ with respect to $\mathbf{u}$. Since the calculation of the normalized gradient vector $\partial H / \partial \mathbf{u}$ is not directly accessible and according to our several numerical applications, we find that the normalized gradient in Eq. (15) considering Eq. (18) can be expressed as

$\frac{\partial H}{\partial u_{i}}=\sqrt{\frac{\partial G}{\partial y_{i}}}, \quad i=1, \ldots, n$

Eq. (15) at the optimum value of the normalized vector can be written in the following form:

$u_{i}^{*}= \pm \beta_{t} \sqrt{\frac{\left|\frac{\partial G}{\partial y_{i}}\right|}{\sum_{j=1}^{n}\left|\frac{\partial G}{\partial y_{j}}\right|}}, \quad i=1, \ldots, n$

where the sign of \pm depends on the sign of the derivative, i.e.,

$\frac{\partial G}{\partial y_{i}}>0 \Longleftrightarrow u_{i}^{*}>1$ and $\frac{\partial G}{\partial y_{i}}<0 \Longleftrightarrow u_{i}^{*}<1, \quad i=1, \ldots, n$

\subsection{Statistical developments}

According to the reliability index definition of Hasofer-Lind (1974), an iso-probabilistic transformation can be carried out between the physical space and normalized one (Fig. 2). The target reliability index that corresponds to the failure probability, is numerically computed as follows

$P_{f} \approx \Phi\left(-\beta_{t}\right)$ or $\beta_{t} \approx-\Phi^{-1}\left(P_{f}\right)$

where $\Phi(\cdot)$ is the standard Gaussian cumulated function given as follows:

$\Phi(Z)=\frac{1}{\sqrt{2 \pi}} \int_{-\infty}^{Z} e^{-\frac{z^{2}}{2}} d z$

Using the basic definition of Hasofer-Lind reliability index (22), we consider a simple normalized mapping transformation for the five most commonly used probabilistic distributions (normal, lognormal, uniform, Gumbel and Weibull distributions).

\subsubsection{OSF for normal distribution}

In general, when considering the normal distribution law, the transformation between the physical space (or $\mathbf{x}$-space) and the normalized space (or $\mathbf{u}$-space) is defined by

$y_{i}=x_{i}+\sigma_{i} u_{i}, \quad i=1, \ldots, n$

Considering that the random variable mean values $m_{i}$ are presented by deterministic design variable $x_{i}$. This way the design point can be defined as:

$y_{i}=S_{f_{i}} \cdot x_{i}, \quad i=1, \ldots, n$

Note that using Eqs. (23a) and (23b), the optimum safety factor associated with $u_{i}^{*}$ can be written as

$S_{f_{i}}=1+\gamma_{i} \cdot u_{i}^{*}, \quad i=1, \ldots, n$

where the variance coefficient $\gamma_{i}$ relating the mean $m_{i}$ and standarddeviation $\sigma_{i}$ equals to: $\gamma_{i}=\sigma_{i} / m_{i}$.

\subsubsection{OSF for lognormal distribution}

For lognormal distribution law, the transformation is defined by

$y_{i}=e^{\mu_{i}+\zeta_{i} u_{i}}, \quad i=1, \ldots, n$

and the normalized variable $u_{i}$ is given by 


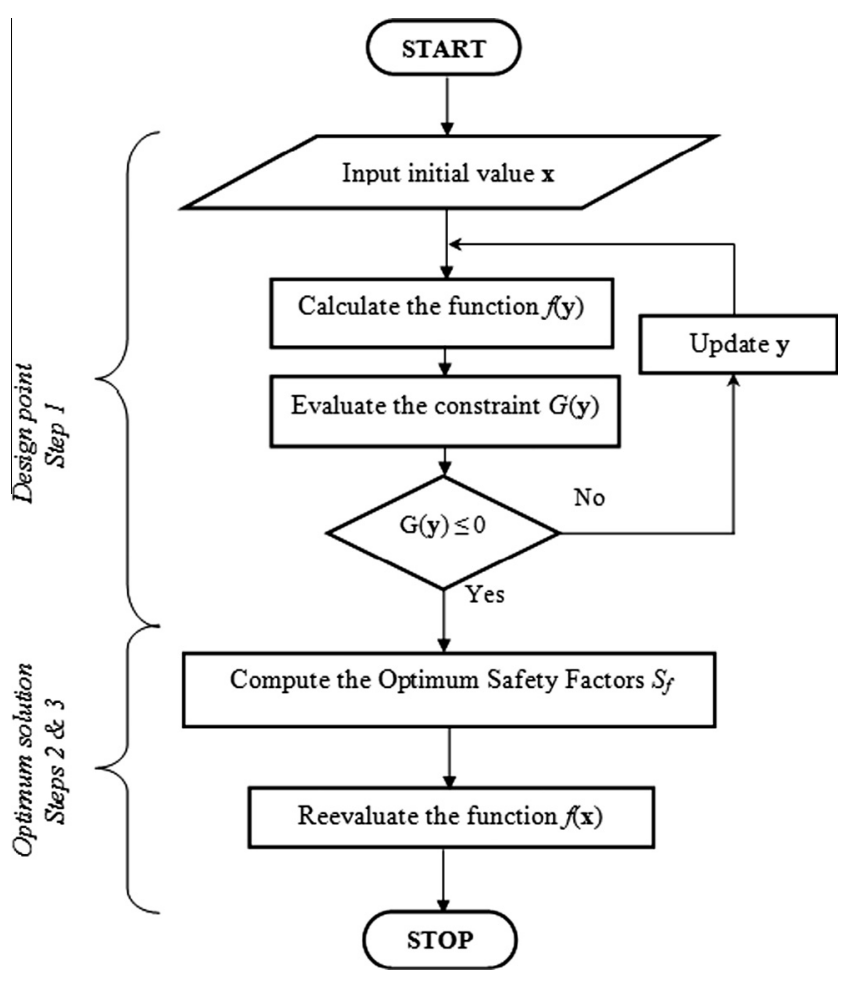

Fig. 4. Flowchart of OSF approach.

$u_{i}=\frac{\ln \left(y_{i}\right)-\ln \left(\frac{m_{i}}{\sqrt{1+\gamma_{i}^{2}}}\right)}{\sqrt{\ln \left(1+\gamma_{i}^{2}\right)}}=\frac{\ln \left(\frac{y_{i} \sqrt{1+\gamma_{i}^{2}}}{m_{i}}\right)}{\sqrt{\ln \left(1+\gamma_{i}^{2}\right)}}, \quad i=1, \ldots, n$

When considering the lognormal distribution and assuming a single limit state failure scenario $G(\mathbf{y}) \leqslant 0$, the equation for the optimum safety factor can be written in a way similar to that presented in Section 6.2.1. Hence, we get the optimum safety factors in terms of the optimum values of the normalized variables $u_{i}^{*}$ (see Eq. (20)), and the equation for the optimum safety factors can be written as

$S_{f_{i}}=\frac{1}{\sqrt{1+\gamma_{i}^{2}}} \exp \left(\sqrt{\ln \left(1+\gamma_{i}^{2}\right)} \cdot u_{i}^{*}\right), \quad i=1, \ldots, n$

\subsubsection{OSF for uniform distribution}

For uniform distribution law, the transformation is defined by

$y_{i}=a+(b-a) \Phi\left(u_{i}\right), \quad i=1, \ldots, n$

and the normalized variables $u_{i}$ are given by

$u_{i}=\Phi^{-1}\left(\frac{y_{i}-a}{b-a}\right), \quad i=1, \ldots, n$

where $a$ and $b$ are the end (bound) values of the interval for $y_{i}$, and $\Phi$ is the distribution function. The mean value $m_{i}$ is given by

$m_{i}=x_{i}=\frac{a+b}{2}, \quad i=1, \ldots, n$

and the standard deviation $\sigma_{i}$ by

$\sigma_{i}=\frac{b-a}{\sqrt{12}}, \quad i=1, \ldots, n$

From Eq. (27c \& d), we get:

$a=x_{i}-\sqrt{3} \sigma_{i}, \quad i=1, \ldots, n$ and

$b=x_{i}+\sqrt{3} \sigma_{i}, \quad i=1, \ldots, n$

Using Eqs. (23b) and (27), we get the following expression for the optimum safety factor corresponding to the optimum value of the normalized variable $u_{i}^{*}$ :

$S_{f_{i}}=1-\sqrt{3} \gamma_{i}\left(1-2 \Phi\left(u_{i}^{*}\right)\right), \quad i=1, \ldots, n$

\subsubsection{OSF for Weibull distribution}

For Weibull distribution law, the transformation is defined by

$y_{i}=v\left[-\ln \left(\Phi\left(-u_{i}\right)\right)\right]^{1 / k}$

with $\Phi\left(-u_{i}\right)=1-\Phi\left(u_{i}\right), k$ : shape factor $>0, v$ : measure factor $>0$, the mean is given by:

$m_{i}=v \Gamma\left(1+\frac{1}{k}\right) \Rightarrow v=\frac{m_{i}}{\Gamma\left(1+\frac{1}{k}\right)}$

and the standard-deviation is given by:

$\sigma_{i}^{2}=v^{2}\left[\Gamma\left(1+\frac{2}{k}\right)-\Gamma^{2}\left(1+\frac{1}{k}\right)\right] \Longleftrightarrow \gamma_{i}=\sqrt{\frac{\Gamma\left(1+\frac{2}{k}\right)}{\Gamma^{2}\left(1+\frac{1}{k}\right)}-1}$

where $\Gamma(a)=\int_{0}^{\infty} x^{a-1} e^{-z} d z$ or a factorial form $\Gamma(a)=(a-1)$ ! for integers.

This way the equation of optimum safety factor can be written as:

$S_{f_{i}}=\frac{1}{\Gamma\left(1+\frac{1}{k}\right)}\left[-\ln \left(\Phi\left(-u_{i}^{*}\right)\right)\right]^{1 / k}$

\subsubsection{OSF for Gumbel distribution}

For Gumbel distribution law, the transformation is defined by

$y_{i}=v-\frac{1}{\alpha} \ln \left[-\ln \left(\Phi\left(u_{i}\right)\right)\right]$

where the mean is given by:

$m_{i}=v+\left(\frac{0.577}{\alpha}\right) \Rightarrow v=m_{i}-\left(\frac{0.577}{\alpha}\right)$

and the standard-deviation is given by:

$\sigma_{i}=\frac{\pi}{\sqrt{6} \alpha} \Rightarrow \alpha=\frac{\pi^{2}}{6 \sigma_{i}^{2}}$

Using (23b), (31b \& c), Eq. (31a) can be written as

$S_{f_{i}}^{1,2}=\frac{1}{2}\left(1 \pm \sqrt{1-\frac{24}{\pi^{2}} \gamma_{i}^{2} y_{i}\left(0.577-\ln \left[-\ln \left(\Phi\left(u_{i}^{*}\right)\right)\right]\right)}\right)$

Using these safety factors, we can satisfy the required reliability level and significantly reduce the complexity of the problem.

\subsection{OSF algorithm}

The Optimum Safety Factor (OSF) algorithm can be easily implemented in three principal steps (Fig. 4):

1. Determine the design point: we consider the most active constraint as a limit state function $G(\mathbf{y})$. The optimization problem is to minimize the objective function subject to the limit state and the deterministic constraints. The resulting solution is considered as the most probable failure point and is termed the design point.

2. Compute the safety factors: in order to compute these factors using Eqs. (24), (26), (28), (30) and (32), a sensitivity analysis of the limit state function with respect to all variables is 


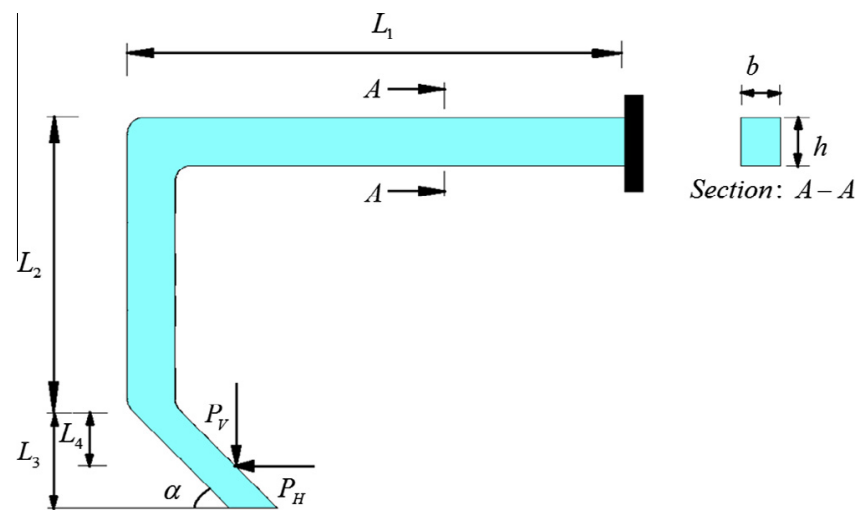

Fig. 5. A schematic drawing of the chisel plough shank with acting forces.

required. When the number of the deterministic variables is equal to that of the random ones, there is no need for additional computational cost when the gradient calculation is carried out during the optimization process of the design point. If the number of the deterministic variables is different from that of the random ones, we need only to evaluate the sensitivity of the limit state function with respect to those random variables that are not common with the deterministic.

3. Calculate the optimal solution: in the last step, we include the values of the safety factors in the computation of the values of the design variables and then determine the optimum design of the structure.

\section{Numerical application}

\subsection{Problem description}

The studied chisel plough illustrated in Fig. 5, can be used primarily to realize the weed control, the seedbed preparation and other secondary tillage operations. According to the deterministic design studies, the designer proposes a global safety factor on the yield stress value. The RBDO solution can reduce the structural weight in uncritical regions. It does not only provide an improved design but also a higher level of confidence in the design. For example, the allowable stress design methods use a safety factor to compute the allowable stresses in members from the ultimate stress, and a successful design ensures that the stresses caused by the values of the loads do not exceed the allowable stresses $\sigma_{w}=\sigma_{y} / S_{f}$ where $S_{f}$ is the global safety factor. The values of the proposed safety factors principally depend on the engineering experience that may lead to low reliability level or to high cost. In this application, we consider that the studied parameters are presented by probabilistic characteristics.

To determine the distribution types of soil mechanical properties, we consider (32) samples of soil mechanical properties for different soil types presented in Appendix. First, we model the histograms and the probability density functions of different studied properties as illustrated in Figs. 6a-6e.

Next, we determine the corresponding distribution types according to the shape of the histogram and parameters as illustrated in Table 1. Here, $\mu$ and $\xi$ are the shape and scale parameters of a lognormal distribution, $k$ and $\lambda$ are, respectively, the scale and shape parameters of a Weibull distribution and $\eta$ is the scale parameter of a exponential distribution.

The distributions of soil forces are established only for a shank chisel plow, Figs. 7a and 7b. In this study, we select a simple chisel plow containing shovels as narrow tines in order to calculate the soil-tool forces in applying McKyes and Ali's model. Tool and operating parameters of a shank chisel plow are presented in Table 2.

Therefore, the horizontal and vertical forces follow lognormal distribution laws and theirs probabilistic characteristics are presented in Table 3.

The performance criterion, related to the mechanical resistance of tillage machines is determined by the difference between the yield stress and the maximum stress. Therefore, the limit state function that defined the safe region can be written using the following equation:

$G(\mathbf{x}, \mathbf{y})=\sigma_{\max }-\sigma_{y} \leq 0$
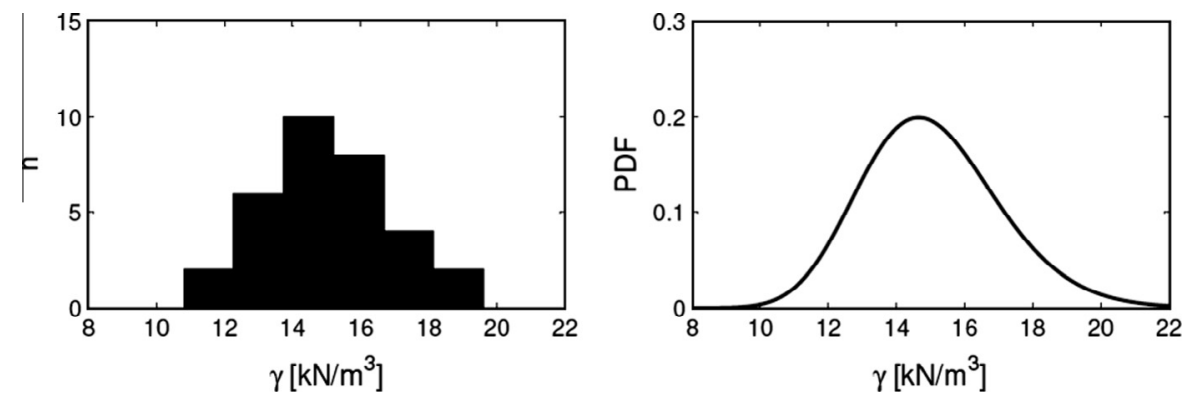

Fig. 6a. Histogram and probability density function of soil density.
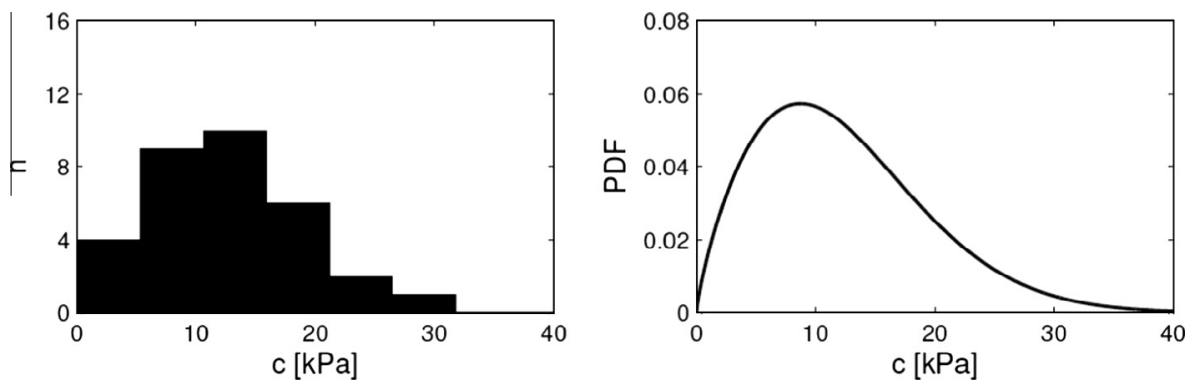

Fig. 6b. Histogram and probability density function of soil cohesion. 

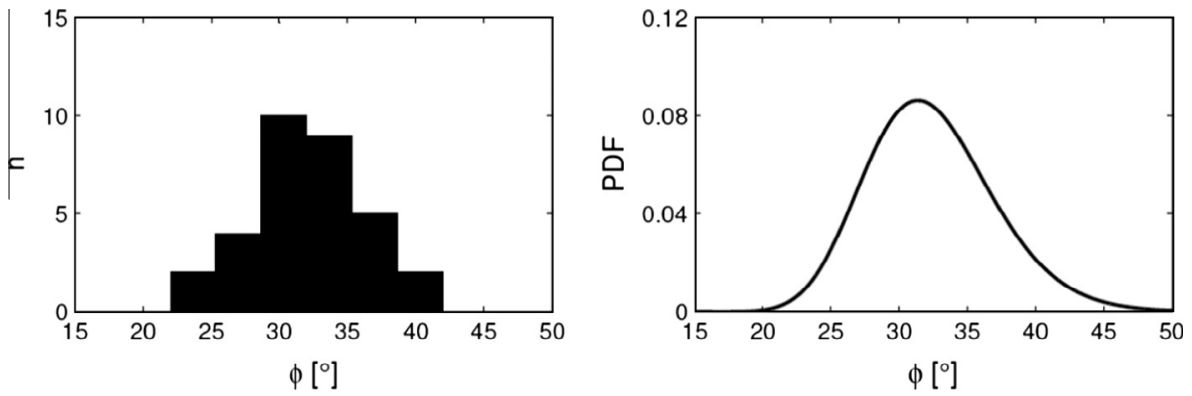

Fig. 6c. Histogram and probability density function of internal friction angle.
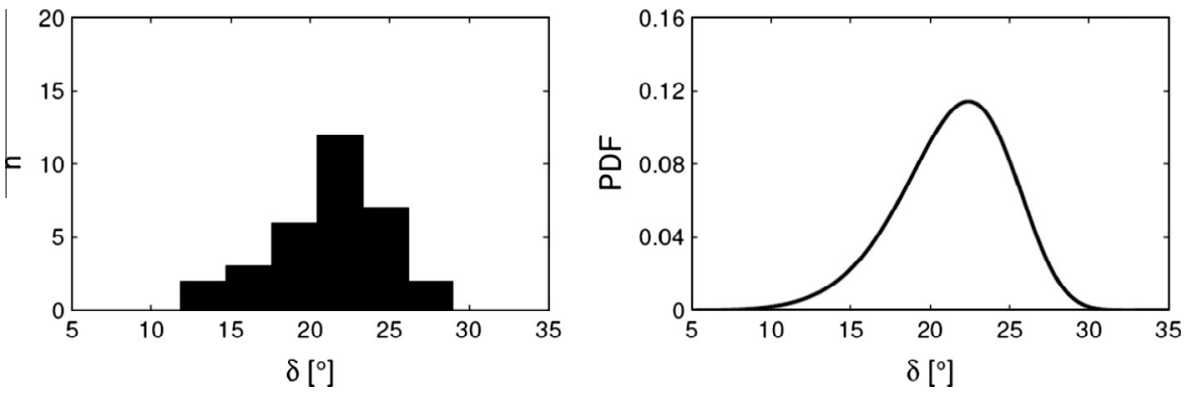

Fig. 6d. Histogram and probability density function of external friction angle.
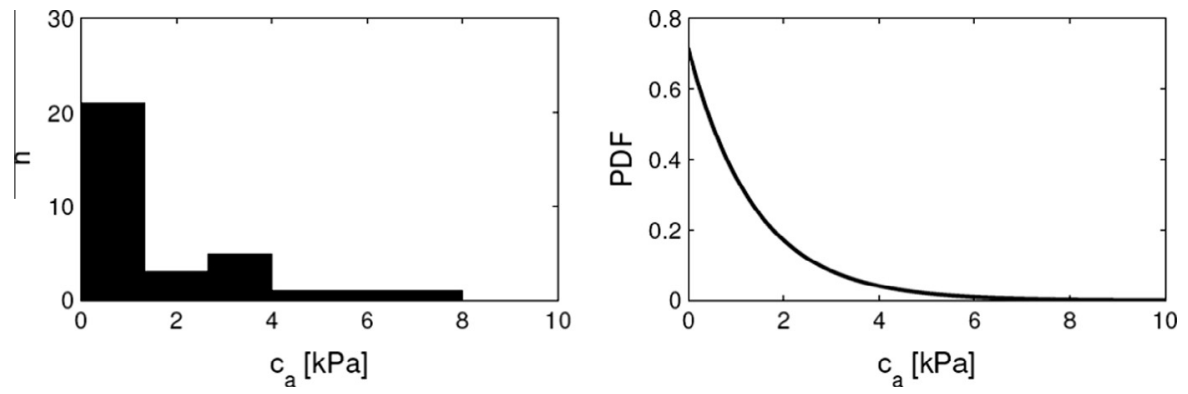

Fig. 6e. Histogram and probability density function of soil-tool adhesion.

Here, $\mathbf{x}$ is the vector of deterministic variables and $\mathbf{y}$ is the vector of random variables, $\sigma_{y}$ is the yield stress $\left(\sigma_{y}=235 \mathrm{MPa}\right)$ and $\sigma_{\max }$ is the maximum stress being calculated analytically using the strength materials rules and given by:

$\sigma_{\max }=\frac{6}{b \cdot h^{2}}\left[\left(L_{2}+L_{4}\right) \cdot P_{H}+\frac{L_{4}}{\tan (\alpha)} \cdot P_{V}\right]+\frac{1}{b \cdot h} \cdot P_{H}$

The limit state function of the simplified shank model, illustrated in Fig. 5, is a function of the following variables as:

$G(\mathbf{x}, \mathbf{y})=f\left(P_{H}, P_{V}, \alpha, b, h, L_{2}, L_{4}\right)$

Table 4 presents the input geometrical parameters of the studied shank. In the next sections, we study two cases of design optimization under uncertainties. The first case corresponds to the randomness of the loading while the second one shows the consideration importance of the randomness of both geometry and loading.

\subsection{Optimum design under random loading}

Considering given values of the horizontal and vertical forces as: $P_{H}=2.463(\mathrm{kN})$ and $P_{V}=1.032(\mathrm{kN})$, the corresponding maximum stress value (using Eq. (34)) of the initial point equals to:
Table 1

Probabilistic characteristics of soil engineering properties.

\begin{tabular}{lll}
\hline Variable & Type & Distribution parameters \\
\hline$\gamma\left(\mathrm{kN} / \mathrm{m}^{3}\right)$ & Lognormal & $\mu=2.703, \xi=0.135$ \\
$c(\mathrm{kPa})$ & Weibull & $k=13.924, \lambda=1.777$ \\
$\phi\left({ }^{\circ}\right)$ & Lognormal & $\mu=3.467, \xi=0.146$ \\
$\delta\left({ }^{\circ}\right)$ & Weibull & $k=22.909, \mu=7.047$ \\
$c_{a}(\mathrm{kPa})$ & Exponential & $\eta=0.716$ \\
\hline
\end{tabular}

$\sigma_{\max }=63.99(\mathrm{MPa})$. The global safety factor of this point can be calculated as:

$S_{f}=\frac{\sigma_{y}}{\sigma_{\max }}$

Using this equation, we get that the global safety factor on the limit state equals to: $S_{f}=3.67$. Here, we should estimate the reliability level of this structure using Eq. (3).

Considering a two variable problem, the optimization process using ANSYS software (First Order Method) leads to the coordinates of the design point or so-called MPP (Most Probable failure Point) and the actual point (see Table 5). The reliability index considering the force variability of the studied structure equals to: 

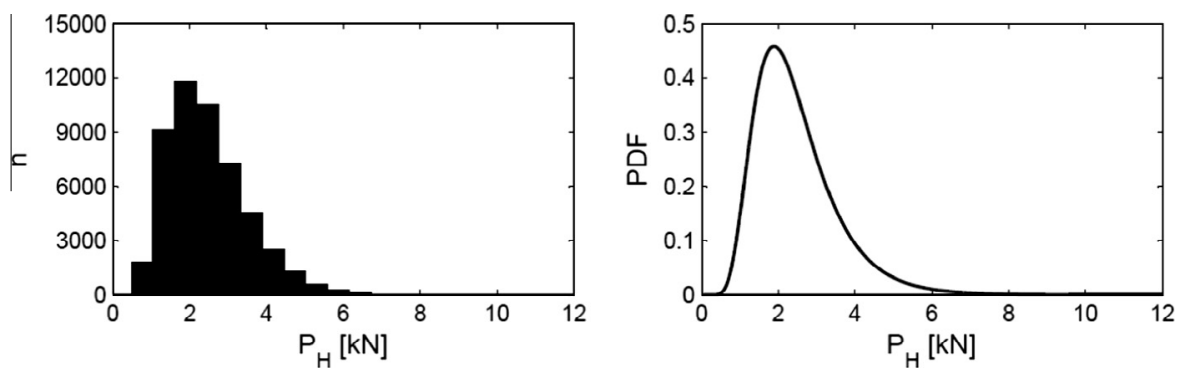

Fig. 7a. Histogram and probability density function of horizontal force.
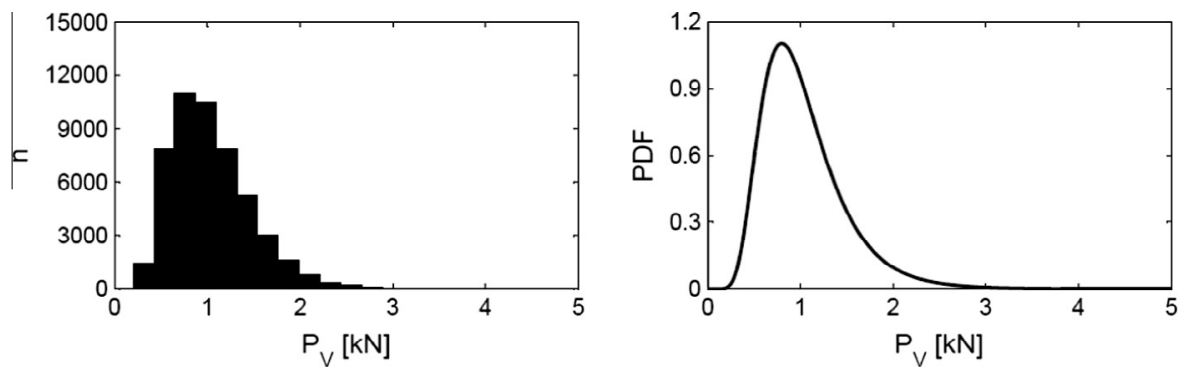

Fig. 7b. Histogram and probability density function of vertical force.

Table 2

Tool and operating parameters of the studied shank.

\begin{tabular}{llll}
\hline$\alpha$ & $d$ & $w$ & $v$ \\
\hline $45^{\circ}$ & $250 \mathrm{~mm}$ & $50 \mathrm{~mm}$ & $6 \mathrm{~km} / \mathrm{h}$ \\
\hline
\end{tabular}

Table 3

Probabilistic characteristics of tillage forces.

\begin{tabular}{lllll}
\hline $\begin{array}{l}\text { Force } \\
\text { type }\end{array}$ & $\begin{array}{l}\text { Distribution } \\
\text { type }\end{array}$ & $\begin{array}{l}\text { Distribution } \\
\text { parameters }\end{array}$ & $\begin{array}{l}\text { Mean } \\
\text { value }\end{array}$ & $\begin{array}{l}\text { Standard- } \\
\text { deviation }\end{array}$ \\
\hline$P_{H}(\mathrm{kN})$ & Lognormal & $\mu=0.815, \xi=0.421$ & 2.463 & 1.044 \\
$P_{V}(\mathrm{kN})$ & Lognormal & $\mu=-0.052, \xi=0.415$ & 1.032 & 0.427 \\
\hline
\end{tabular}

Table 4

Input geometrical parameters of the studied shank.

\begin{tabular}{llllll}
\hline$L_{1}$ & $L_{2}$ & $L_{3}$ & $L_{4}$ & $b$ & $h$ \\
\hline $600 \mathrm{~mm}$ & $350 \mathrm{~mm}$ & $150 \mathrm{~mm}$ & $75 \mathrm{~mm}$ & $32 \mathrm{~mm}$ & $58 \mathrm{~mm}$ \\
\hline
\end{tabular}

Table 5

Reliability analysis of the studied shank.

\begin{tabular}{lll}
\hline Parameters & Actual solution & Design point \\
\hline$P_{H}(\mathrm{kN})$ & 2.463 & 8.907 \\
$P_{V}(\mathrm{kN})$ & 1.032 & 4.560 \\
$\sigma_{\max }(\mathrm{MPa})$ & 63.99 & 234.91 \\
$\beta$ & 4.99 & \\
$P_{f}$ & $3 \times 10^{-7}$ & \\
\hline
\end{tabular}

$\beta=4.99$ that corresponds to probability of failure $P_{f} \approx 3 \times 10^{-7}$ using Eq. (22). In fact, the safety factor is generally integrated to the yield stress or the loads. However, a sensitivity analysis can be helpful to determine the role (influence) of each parameter. Therefore, the proposition of the safety factor without any sensitivity analysis and statistical design may lead to expensive or not reliable structures. When comparing our study relative to the nuclear
Table 6

RBDO of the studied shank.

\begin{tabular}{lll}
\hline Parameters & Optimum solution & Design point \\
\hline$P_{H}(\mathrm{kN})$ & 3.420 & 8.907 \\
$P_{V}(\mathrm{kN})$ & 4.141 & 4.560 \\
$\sigma_{\max }(\mathrm{MPa})$ & 100.16 & 234.91 \\
$\beta$ & 3.00 & \\
$P_{f}$ & $1 \times 10^{-3}$ & \\
\hline
\end{tabular}

Table 7

Sensitivity analysis of the maximum stress function.

\begin{tabular}{lll}
\hline Random parameters & Sensitivity functions & Sensitivity values \\
\hline Geometry & $\frac{\partial \sigma_{\max }}{\partial b}$ & -1.999 \\
& $\frac{\partial \sigma_{\max }}{\partial h^{2}}$ & -2.181 \\
$\frac{\partial \sigma_{\max }}{\partial L_{1}}$ & 0.000 \\
$\frac{\partial \sigma_{\max }}{\partial L_{2}}$ & 0.1373 \\
& $\frac{\partial \sigma_{\max }}{\partial L_{3}}$ & 0.000 \\
& $\frac{\partial \sigma_{\max }}{\partial L_{4}}$ & 0.1948 \\
& $\frac{\partial \sigma_{\max }}{\partial \alpha_{1}}$ & -0.1501 \\
& $\frac{\partial \sigma_{\max }}{\partial P_{H}}$ & 0.02423 \\
Loading & $\frac{\partial \sigma_{\max }}{\partial P_{V}}$ & 0.00418 \\
\hline
\end{tabular}

and spatial ones, we note that the nuclear and spatial studies necessitate very small failure probability, the failure probability should be: $P_{f} \in\left[10^{-6}-10^{-8}\right]$ that corresponds to a reliability index $\beta \epsilon$ [4.75-5.6] however in structural studies, the failure probability should be: $P_{f} \in\left[10^{-3}-10^{-5}\right]$ that corresponds to a reliability index $\beta \in[3-4.25]$. Therefore, we should improve the design reliability to be: $\beta_{t}=3$ using the recent technology of RBDO based on our OSF developments. Since the loads presented by the horizontal and vertical forces follow the lognormal distribution law, we use Eq. (26) to compute analytically the optimum safety factors of the loads.

Table 6 shows the coordinates of the optimum solution points of the RBDO technology. 
Table 8

RBDO of the studied shank under geometry and loading uncertainty.

\begin{tabular}{|c|c|c|c|c|c|}
\hline Random parameters & Distribution laws & OSF $S_{f_{i}}$ & Normalized variable $u_{i}$ & Design point $y_{i}$ & Optimum solution $x_{i}$ \\
\hline$b(\mathrm{~mm})$ & Uniform & 0.835 & -1.958 & 31.687 & 37.917 \\
\hline$h(\mathrm{~mm})$ & Uniform & 0.835 & -2.046 & 57.673 & 69.077 \\
\hline$L_{2}(\mathrm{~mm})$ & Normal & 0.949 & 0.5133 & 350.47 & 332.48 \\
\hline$L_{4}(\mathrm{~mm})$ & Weibull & 1.303 & 0.6114 & 75.174 & 57.6716 \\
\hline$\alpha^{\circ}$ & Gumbel & 0.879 & -0.5366 & 44.914 & 51.1 \\
\hline$P_{H}(\mathrm{kN})$ & Lognormal & 1.005 & 0.2156 & 9.2501 & 9.203 \\
\hline$P_{V}(\mathrm{kN})$ & Lognormal & 0.957 & 0.0895 & 1.3248 & 1.383 \\
\hline$\sigma_{\max }(\mathrm{MPa})$ & & & & 234.89 & 124.73 \\
\hline $\operatorname{Vol}\left(\mathrm{mm}^{3}\right)$ & & & & $2.01 \times 10^{6}$ & $2.83 \times 10^{6}$ \\
\hline$\beta$ & & & & 3.00 & \\
\hline$P_{f}$ & & & & $1 \times 10^{-3}$ & \\
\hline
\end{tabular}

\subsection{Optimum design under random geometry and loading}

Here, we consider the randomness of both geometry and loading parameters. In order to show the influence of different parameters on the maximum stress values, we perform a sensitivity analysis of the maximum stress relative to the geometry and loading. Table 6 defines the sensitivities (derivatives) of the maximum stress with respect to all parameters (geometry and loading). We note that the derivatives with respect to both parameters $L_{1}$ and $L_{3}$ equal to zeros.

Here, we can ignore the influence of these two parameters on the maximum stress values. Therefore, we deal with four geometry parameters $\left(b, h, L_{2}, L_{4}\right.$ and $\alpha$ ) and with two loading parameters $\left(P_{H}\right.$ and $\left.P_{V}\right)$. Table 8 presents the RBDO results when applying the developed OSF equations for different distribution laws. Table 1 presents the probabilistic data. For both horizontal and vertical forces, the distribution laws (lognormal), the means and the standard-deviations are presented in Table 3 as given data. To compute the OSF, we use Eq. (26). Since we apply the new strategy on the randomness of the geometry parameters with object of demonstrating its efficiency, the standard-deviations of the random geometry parameters are considered as proportional value of the means $\left(\sigma_{i}=0.1 m_{i}\right)$ for simplicity.

Furthermore, we consider that the length dimension $L_{2}$, the section shank dimensions ( $b$ and $h$ ), the depth dimension $L_{4}$ and the angle parameter $a$ follow respectively the normal, uniform, Weibull and Gumbel distributions. Here, we respectively use Eqs. (24), (28), (30) and (32) to compute the OSF of these different geometry parameters. The sensitivity analysis presented in Table 7 shows that the geometry parameters have a bigger influence than the loading parameters while in the classical design process, we propose the safety factor integration into the loading. We conclude that using the optimization process based on the sensitivity analysis, we efficiently control structural designs to satisfy a required reliability level and an acceptable cost.

\section{Conclusions and perspectives}

In this paper, we develop an efficient methodology that can lead to optimum designs under uncertainties. Here, the developed method controls the structural reliability levels for complex studies. The basic idea of the developed strategy is to find structural sensitivity values with object of determining the influence of each random parameter. An efficient application on the chisel shank plough under the uncertainties on the soil tillage forces is detailed. Here, the tillage forces are calculated in accordance with analytical model of McKyes and Ali. The advantage of the RBDO using OSF is to define the best compromise between cost and safety. Furthermore, we show that the classical design considering the uncertainty on the loading parameter may not lead to economic or reliable structures. Therefore, we demonstrate that the sensitivity analysis and developed RBDO strategy can provide designers with the different qualities (reliability, performance, cost, ...).

\section{Acknowledgements}

This research is an extension of a $\mathrm{PhD}$ thesis of $\mathrm{Mr}$. Abo Al-kheer which is defended at INSA de Rouen, France (2010).

\section{Appendix A}

A.1. Statistical data of 32 samples

\begin{tabular}{|c|c|c|c|c|c|}
\hline No. & $\gamma\left(\mathrm{kN} / \mathrm{m}^{3}\right)$ & $c(\mathrm{kPa})$ & $\phi\left({ }^{\circ}\right)$ & $\delta\left(^{\circ}\right)$ & $c_{a}(\mathrm{kPa})$ \\
\hline 1 & 14.70 & 04.60 & 37.5 & 11.9 & 0.01 \\
\hline 2 & 10.80 & 00.10 & 34.0 & 14.4 & 3.29 \\
\hline 3 & 14.61 & 02.26 & 35.0 & 15.2 & 2.20 \\
\hline 4 & 15.70 & 07.19 & 35.0 & 15.9 & 2.70 \\
\hline 5 & 14.34 & 06.30 & 37.3 & 22.0 & 2.50 \\
\hline 6 & 11.00 & 11.90 & 29.8 & 17.2 & 0.00 \\
\hline 7 & 14.50 & 06.00 & 28.8 & 18.3 & 0.00 \\
\hline 8 & 13.20 & 23.00 & 33.1 & 18.8 & 0.00 \\
\hline 9 & 14.12 & 08.90 & 35.0 & 18.8 & 2.31 \\
\hline 10 & 16.19 & 12.80 & 32.0 & 19.8 & 0.18 \\
\hline 11 & 13.05 & 16.70 & 35.0 & 19.9 & 0.21 \\
\hline 12 & 16.38 & 15.50 & 22.0 & 20.0 & 0.29 \\
\hline 13 & 13.73 & 06.00 & 23.3 & 21.6 & 0.35 \\
\hline 14 & 14.02 & 23.00 & 27.1 & 22.0 & 0.31 \\
\hline 15 & 16.98 & 10.50 & 30.8 & 22.0 & 3.25 \\
\hline 16 & 16.38 & 31.75 & 42.0 & 22.0 & 5.27 \\
\hline 17 & 14.02 & 12.10 & 30.2 & 22.3 & 3.22 \\
\hline 18 & 15.30 & 13.30 & 36.5 & 22.4 & 3.21 \\
\hline 19 & 15.79 & 20.50 & 35.0 & 23.0 & 0.00 \\
\hline 20 & 17.66 & 05.00 & 38.0 & 23.0 & 0.00 \\
\hline 21 & 19.62 & 10.20 & 32.5 & 23.1 & 0.00 \\
\hline 22 & 19.00 & 20.40 & 31.8 & 23.3 & 0.00 \\
\hline 23 & 14.71 & 13.90 & 30.3 & 23.5 & 0.60 \\
\hline 24 & 14.91 & 15.50 & 39.3 & 23.8 & 0.00 \\
\hline 25 & 15.30 & 15.30 & 32.6 & 24.0 & 0.00 \\
\hline 26 & 15.01 & 06.70 & 31.4 & 24.1 & 0.00 \\
\hline 27 & 14.62 & 11.70 & 29.2 & 24.5 & 0.00 \\
\hline 28 & 12.80 & 07.00 & 31.4 & 24.7 & 0.00 \\
\hline 29 & 13.50 & 17.00 & 37.6 & 25.0 & 0.00 \\
\hline 30 & 13.23 & 16.00 & 26.6 & 23.2 & 0.00 \\
\hline 31 & 16.98 & 11.70 & 27.4 & 27.3 & 6.66 \\
\hline 32 & 18.05 & 19.50 & 28.4 & 29.0 & 8.00 \\
\hline
\end{tabular}




\section{References}

Abo Al-kheer, A., 2010. Integrating the Concepts of Optimization and Reliability in the Design of Agricultural Machines. PhD Thesis. November 19, Rouen National Institute of Applied Sciences, France.

Abo Al-kheer, A., Kharmanda, G., El Hami, A., Mouazen, A., 2011. Estimating the variability of tillage forces on a chisel plough shank by modeling the variability of tillage system parameters. J. Comput. Electron. Agric. 78 (1), 61-70.

Arora, J.S., 1989. Introduction to Optimum Design. McGraw-Hill, New York, NY.

Du, X., Chen, W., 2004. Sequential optimization and reliability assessment method for efficient probabilistic design. J. Mech. Des. 126, 225-233.

Feng, Y.S., Moses, F., 1986. A method of structural optimization based on structural system reliability. J. Struct. Mech. 14, 437-453.
Haftaka, R.T., Gurdal, Z., 1991. Elements of Structural Optimization. Kluwer Academic Publications, Dordrecht, Netherlands.

Hasofer, A.M., Lind, N.C., 1974. An exact and invariant first order reliability format. J. Eng. Mech. ASCE EM1 100, 111-121.

Kharmanda, G., Sharabaty, S., Ibrahim, H., Makhloufi, A.H., El Hami, A. 2009. Reliability-based design optimization using semi-numerical methods for different engineering application. Int. J. CAD/CAM 9, 1-16.

Lemaire, M., 2005. Fiabilité des structures, Paris. HERMES-LAVOISIER, pp. 506.

Mckyes, E., Ali, O.S., 1977. The cutting of soil by narrow blades. J. Terrramech. 14 (2), 43-58.

Reece, A.R., 1965. The fundamental equations of earth-moving mechanics. Proceedings of the Symposium on Earth Moving Machinery, vol. 179. Institute of Mechanical Engineering, pp. 8-14. 